\title{
Constraining $S$-wave velocity using Rayleigh wave ellipticity from polarization analysis of seismic noise
}

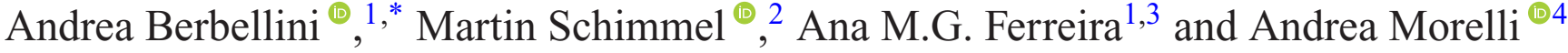 \\ ${ }^{1}$ Department of Earth Sciences, Faculty of Maths \& Physical Sciences, University College London, WC1E6BT, United Kingdom. \\ Email: andrea.berbellini@ingvit \\ ${ }^{2}$ Institut de Ciencies de la Terra Jaume Almera - CSIC, Barcelona, Spain \\ ${ }^{3}$ CERIS, Instituto Superior Tecnico, Universidade de Lisboa, Av. Rovisco Pais 1, 1049-001 Lisboa, Portuga \\ ${ }^{4}$ Istituto Nazionale di Geofisica e Vulcanologia, Sezione di Bologna, Via Donato Creti 12, 40128 Bologna, Italy
}

Accepted 2018 November 29. Received 2018 November 28; in original form 2017 December 15

\begin{abstract}
SUMMAR Y
We develop a new method for measuring ellipticity of Rayleigh waves from ambient noise records by degree-of-polarization (DOP) analysis. The new method, named DOP-E, shows a good capability to retrieve accurate ellipticity curves separated from incoherent noise. In order to validate the method we perform synthetic tests simulating noise in a 1-D earth model. We also perform measurements on real data from Antarctica and Northern Italy. Observed curves show a good fit with measurements from earthquake records and with theoretical ellipticity curves. The inversion of real data measurements for $v_{S}$ structure shows a good agreement with previous models. In particular, the shear-wave structure beneath Concordia station shows no evidence of a significant layer of liquid water at the base of the ice. The new method can be used to measure ellipticity at high frequency and therefore it will allow the imaging of near-surface structure, and possibly of temporal changes in subsurface properties. It promises to be useful to study near-surface processes in a wide range of geological settings, such as volcanoes, fault zones and glaciers.
\end{abstract}

Key words: Crustal imaging; Seismic noise; Seismic tomography; Site effects; Surface waves and free oscillations.

\section{INTRODUCTION}

Rayleigh waves are surface waves with particle motion polarized along a vertical ellipse, typically with a retrograde motion at very shallow depths. The ratio of horizontal and vertical axes of the particle motion ellipse is defined as the ellipticity of Rayleigh waves and under ray theory assumptions (scale length of lateral heterogeneity being large compared to wavelength) it depends only on the local structure beneath the station (Woodhouse 1974; Ferreira \& Woodhouse 2007b; Maupin 2017). Because of this feature it represents a powerful tool for investigating the local structure at the receiver particularly in the cases of poor or uneven seismic network coverage. In the last decade a number of studies measured Rayleigh wave ellipticity from earthquake records (e.g. Ferreira \& Woodhouse 2007a; Tanimoto \& Rivera 2008). In some cases ellipticity measurements have been used to better constrain the upper crust in a joint inversion together with phase and group velocity data (e.g. Lin et al. 2012). A first attempt of retrieving a crustal model from Rayleigh wave ellipticity measurements alone (i.e. without using

\footnotetext{
* now at Istituto Nazionale di Geofisica e Vulcanologia, Sezione di Bologna,
} Via Donato Creti 12, 40128 Bologna, Italy. any other data types) has been performed by Yano et al. (2009) but they found a very strong dependency of the final model on the starting one due to the linearized inversion technique used. Recently more extensive measurements of ellipticity in Northern Italy have been performed by Berbellini et al. (2016); they obtained a new crustal model from ellipticity curves alone using a completely nonlinear inversion technique (Berbellini et al. 2017). Using a similar approach, Attanayake et al. (2017) built a first crustal model of Portugal from ellipticity measurements alone. In both studies, the depth parametrization was based on a priori knowledge of the regions considered (e.g. Moho depth and thickness of the layers from previous studies), which helped to constrain the inversions. In fact, since there is a well-known trade-off between velocity and thickness of a layer (Scherbaum \& Ohrnberger 2003; Hobiger et al. 2013) using a parametrization with thickness fixed to $a$ priori values helps to retrieve reliable $v_{S}$ profiles. In addition to these recent advances on Rayleigh wave ellipticity studies based on earthquake data, $\mathrm{H} / \mathrm{V}$ measurements from ambient vibrations have been used for decades for engineering and shallow geophysics purposes such as soil characterization, estimation of site effects and seismic microzonation (e.g. Fäh et al. 2003; Sánchez-Sesma et al. 2011; Hobiger et al. 2013, Pastén et al. 2016). One of the first methods to measure H/V 
from ambient noise has been introduced by Nakamura (1989). This method, rather empirical, is still largely used because of its stable and reliable results in addition to its simplicity and short time windows needed for a measurement. An extensive European project called SESAME-GEOPSY (http://sesame.geopsy.org/) had the aim of developing tools to implement the Nakamura method for site effect estimation for seismic mitigation. These methods are based on the spectral analysis of ambient noise, often assuming that seismic noise is composed mostly by Rayleigh waves (Haubrich et al. 1963; Tanimoto et al. 2006). Nevertheless, it is known that such $\mathrm{H} / \mathrm{V}$ measurements can be strongly affected, for example by Love waves, which can lead to an overestimation of ellipticity. Several methods have been introduced to enhance ellipticity measurements by separating the contribution of Love and body waves. Hobiger et al. (2009) developed a new single-station measurement technique (named RayDec) that uses the random decrement technique, previously used to measure structural parameters of buildings (such as resonance frequencies and damping parameters), to suppress the contribution of Love waves. Other methods take advantage of dense station arrays, for example by performing $f-k$ analysis on the data to separate the contribution of Rayleigh and Love waves (Poggi et al. 2012). More recently a number of works has been published like Hobiger et al. (2016) and Maranò et al. (2017). Here they use a dense seismic array to analyse the polarity of the incoming waves to determine backazimuth, polarization and ellipticity of Rayleigh waves. The use of a seismic array also avoids the $180^{\circ}$ ambiguity in the backazimuth determination, hence they allow identification of retrograde/prograde polarity. These methods, even if very successful, have the main disadvantage of requiring dense seismic arrays, which limits their applicability to temporary deployments. Also, they use high frequency data $(0.2-10 \mathrm{~Hz})$ and they have been extensively used to study the top few hundreds metres of the subsurface.

The aim of this work is to develop a new single-station method for measuring Rayleigh wave ellipticity from ambient noise records to be used in seismological applications such as shallow crustal imaging and crustal tomography. We use a measurement scheme previously developed by Schimmel \& Gallart (2004) based on the degree-of-polarization (DOP) analysis. This algorithm detects polarized signals through their arbitrary but stable particle motion, which is measured by the DOP. In Schimmel et al. (2011) the DOP has been adapted to select seismogram segments where the particle motion describes a stable ellipse in a vertical plane as expected for Rayleigh waves. Here, we focus on Rayleigh waves and further use this concept, which we now label DOP-E to stress that we are considering elliptically polarized waves to measure the $\mathrm{H} / \mathrm{V}$ ratio only on these parts of the records. As an output we obtain an ellipticity curve as a function of wave period and the corresponding backazimuth of the ambient noise for each period. We validate the DOP-E method using synthetic tests for ambient noise computed on a 1-D earth model and we compare H/V measurements from DOP-E with earthquake-based measurements from recording stations in two different geological settings. Finally, we jointly invert the ellipticity measurements from ambient noise and earthquake records for stations CCD (Concordia, Antarctica) and PRMA (Parma, Northern Italy) and we obtain $v_{S}$ profiles that are compatible with previous results.

\section{MEASUREMENT METHOD}

In the following, we briefly summarize the polarization approach used to identify Rayleigh waves in three-component ambient noise

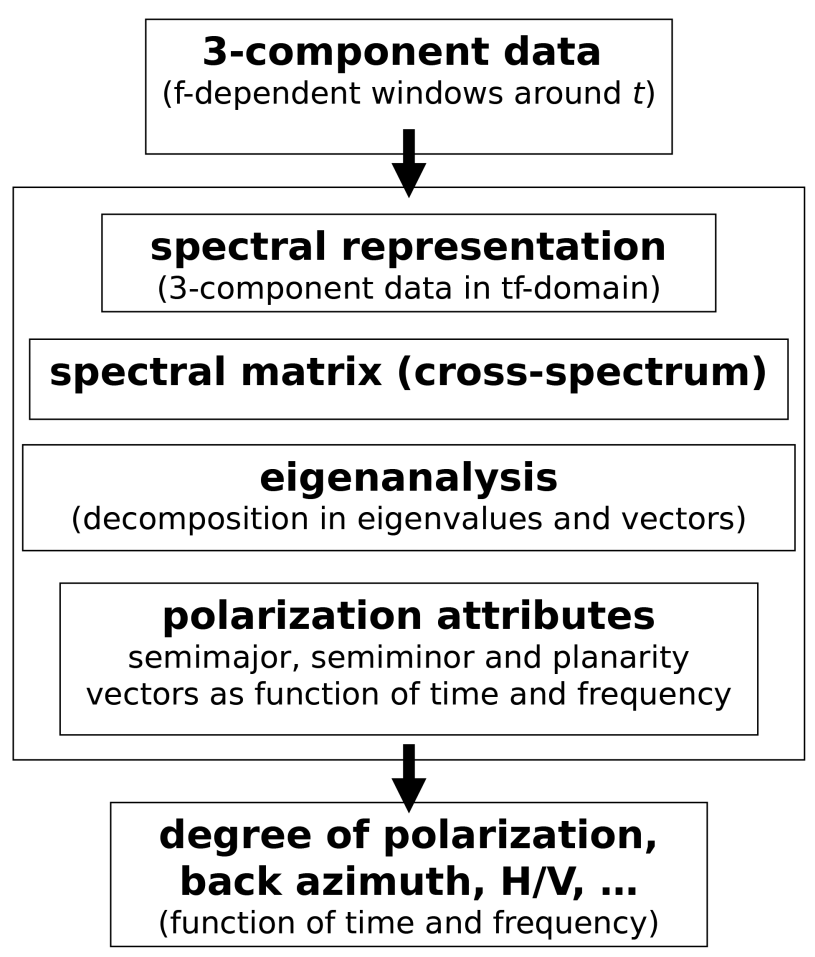

Figure 1. The flowchart illustrates the work flow of the polarization approach. Starting with the three-component seismograms, their spectral representations are determined to then compute the spectral matrix, which after an eigen analysis outputs the DOP, backazimuth and ellipticity as a function of time and frequency.

records. The particle motion ellipse of the identified signals is used to obtain frequency-dependent $\mathrm{H} / \mathrm{V}$ measurements. A work-flow of the measurement scheme is shown in Fig. 1.

\subsection{DOP method}

In Schimmel \& Gallart (2003), signal has been defined as any waveform with arbitrary polarization which remains stable for the duration of the signal. The stability of the polarization state is determined through a moving window analysis on polarization attributes which are extracted from the three-component data. The corresponding stability measure is called DOP and ranges between 0 and 1 , where 1 indicates stable polarization and 0 a randomly changing polarization throughout the moving data window. The DOP is computed from the vector projections of the normalized semi-major and planarity vectors. Semi-major and semi-minor vectors are instantaneous polarization attributes which describe the ellipse that best fits the ground motion. The planarity vector is the vector crossproduct of the semi-major with the semi-minor vector and stands perpendicular on the polarization ellipse. The DOP is a functional of time which can be used to down-weight less polarized signals in filters or to identify signals as the most stable polarized features in seismograms.

This time domain DOP has been extended to the time-frequency domain (Schimmel \& Gallart 2004), where the polarization attributes are determined through a time-frequency dependent eigendecomposition of spectral covariance matrices. The S-transform by Stockwell et al. (1996) is used to achieve the time-frequency representation of the data to compute spectral matrices. This procedure involves a sliding time window of Gaussian shape which is proportional to the analysis period. The width of this window 
defines the time-frequency resolution. Following this approach the instantaneous polarization attributes are determined as a function of time and frequency. They are used in full analogy to the time domain DOP to obtain the time-frequency DOP. Thus, the determination of the time-frequency DOP involves two different sliding data windows: (i) A Gaussian window for the transformation into the time-frequency domain to determine the polarization attributes as a function of time and frequency and (ii) a boxcar window to determine the stability of the polarization, that is the DOP. The temporal width of the Gaussian window is typically $2-5$ periods at one standard deviation while the window for the DOP determination is much shorter. It can be adjusted according to the expected signal length and time duration over which noise shows no stable polarization. That is the boxcar window should be sufficiently long to avoid that parts of noise is detected as stable polarized features. The approach has been adopted to detect, extract and characterize fundamental mode Rayleigh waves from ambient noise recordings (e.g. Schimmel et al. 2011; Obrebski et al. 2012, among others). For that purpose, the DOP has been modified to identify waves which are robustly polarized with elliptical particle motion in a vertical plane, such as expected for fundamental mode Rayleigh waves. The plane of motion is controlled by the planarity vector, that is for Rayleigh waves this vector should lie in a horizontal plane. The measured DOP is down-weighted as a function of the deviation of the ground motion ellipse from the vertical plane. We use $[\cos (\alpha)]^{8}$ as weighting function, where $\alpha$ is the deviation of the planarity vector from the horizontal plane. This function can be considered as a taper and has been chosen so that it has values that vary smoothly from 1 for $\alpha=0^{\circ}$ to 0 for $\alpha=90^{\circ}$ without down-weighting much DOP values for small deviations in $\alpha$. The exact function plays only a very minor role in our processing and other functions with similar behaviour could have been chosen without changing the results.

The noise records are composed of Rayleigh waves generated by different sources, which may interfere in time or frequency. The decomposition into the time-frequency domain used in our approach, therefore, permits to detect Rayleigh waves from different sources as long as their elliptical polarization is stable within the chosen time-window. Using short time windows enables to detect and use Rayleigh waves from different sources. Other waves, as for instance Love waves, may interfere at certain times and frequencies, and decrease the stability of the elliptical particle motion. In these cases the DOP-E will decrease and the signal might not be used depending on the DOP-E threshold. Using seismic records over several days, weeks or months guarantees that sufficient Rayleigh waves from different backazimuths and frequencies are detected to perform the ellipticity analysis.

\subsection{Extraction of ellipticity}

In this work, we are interested in identifying stable Rayleigh wave signals from ambient noise and to further use the frequencydependent shape of the polarization ellipse to constrain the underlying seismic structure. We identify Rayleigh waves as described in the previous subsection. Note that any tilted or inclined particle motion is indicative of anisotropic structure or the interference with other signals and noise. We therefore do not consider polarized signals with an inclined elliptical motion where the semi-major vector deviates by more than $10^{\circ}$ from the vertical or horizontal direction even if the particle motion is in a vertical plane. The final DOP values obtained are only used for signal selection. That is, Rayleigh waves are identified as a function of frequency through a frequencydependent DOP which is larger than a chosen threshold value. As the minimum permitted DOP gets lower, more less-stable polarized signals are permitted, and consequently more signals are included in the subsequent analysis. A high DOP threshold restricts the analysis to better polarized signals. For selected Rayleigh wave frequency components we determine the ellipticity using the particle motion ellipse which is controlled by the instantaneous semi-major and semi-minor vectors. The vertical and horizontal amplitudes are then used from these vectors to build the frequency dependent ellipticity curve.

\section{METHOD VALIDATION}

\subsection{Ambient noise simulation}

To validate the measurement scheme we simulate one day of ambient noise generated by an oceanic storm in the Northern Atlantic in a 1-D earth model. To do so we pick 300 point sources, randomly distributed on a 2-D normal distribution around the coordinates $57^{\circ} \mathrm{N}$, $31^{\circ} \mathrm{W}$ (Fig. 2). The sources are vertical impulses with random amplitudes (between $10^{12} \mathrm{~N} \mathrm{~m}$ and $10^{16} \mathrm{~N} \mathrm{~m}$ ), very short half-duration $(0.5 \mathrm{~s})$ and random origin time. We compute synthetic seismograms at station PRMA (Northern Italy) up to the 5th overtone with a modal summation approach (Herrmann 2013) and using 1-D model PREM (Dziewonski \& Anderson 1981). We then stack all the synthetic seismograms to obtain simulated ambient noise containing only Rayleigh waves. Real ambient noise also contains Love waves (e.g. Friedrich et al. 1998; Nishida et al. 2008; Gualtieri et al. 2013; Tanimoto et al. 2015) but their generation mechanism is not completely known yet. In order to simulate the contribution of Love waves we contaminate the synthetic ambient noise obtained from vertical sources with waveforms generated by 200 random horizontal double-couple sources with random amplitudes (between $10^{12} \mathrm{Nm}$ and $10^{16} \mathrm{Nm}$ ) located in the same area as the vertical sources. We finally add white noise to all the three-component synthetics with a signal-to-noise ratio of 7.5 (ratio between the absolute amplitude mean of the ambient noise and absolute amplitude mean of the white noise) to simulate uncorrelated, spurious noise. This value has been chosen empirically after a series of synthetic tests (see Figs S1-S5). We evaluate the lowest acceptable signal-to-noise ratio (i.e. the highest level of uncorrelated noise) which the DOP-E can handle by adding different levels of white noise to the synthetic noise obtained from our 300 vertical sources. Even if we found that the minimum signal-to-noise ratio that the technique can handle (Figs S1-S5) is 5, we present illustrative results for a slightly higher value for stability issues.

In this validation test we do not want to simulate a perfectly realistic ambient noise to compare to real data observations (e.g. fully accounting for ocean wave models and how they generate ambient noise as in Gualtieri et al. 2013). We only want to test the ability of our method to obtain an ellipticity curve compatible with expected values, distinguishing ambient noise containing Rayleigh waves from other disturbances such as Love waves and uncorrelated noise. Simulating realistic ambient noise to compare with real observations would require a much more complex approach that is beyond the purpose of this work. 


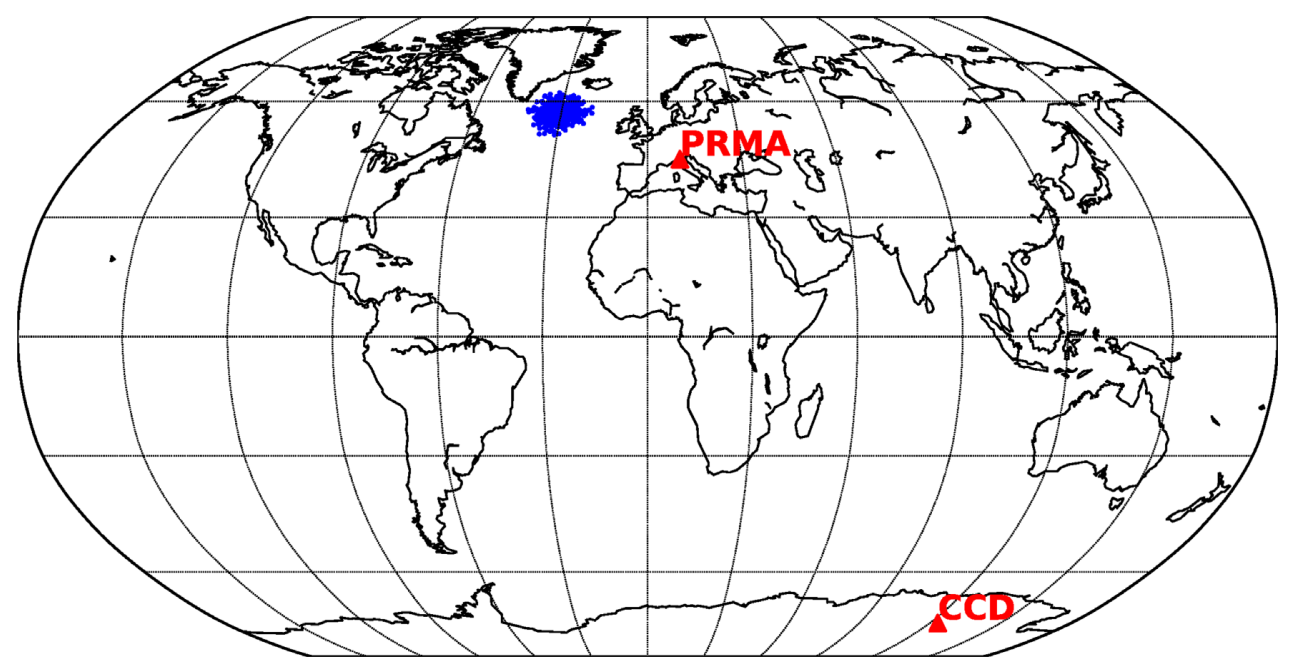

Figure 2. Blue dots: location of the vertical point sources used to simulate ambient noise; red triangle: location of the stations PRMA and CCD.

\subsection{Measurement}

We apply the DOP-E measurement scheme to the synthetic ambient noise in the period range $3-100 \mathrm{~s}$ using the measurement parameters provided in the supplementary material (Table S1). Here we use a narrow Gaussian window of one period within one standard deviation for the time-frequency representation of the data. Longer windows can also be used for instance to stabilize the polarization attributes in noisy data. The DOP window length is inversely proportional to frequency to account for the fact that lower frequency signals should be analysed by larger windows than higher frequency signals. This is in analogy to the frequency-dependent Gaussian window used to compute the time-frequency representation of the data. The DOP window length is defined by a minimum value for the highest analysis frequency and then increased inversely proportional to frequency. Here we use a window length of $8.5 \mathrm{~s}$ for a highest frequency of $0.5 \mathrm{~Hz}$ which means that at $0.1 \mathrm{~Hz}$ this window becomes five times larger. Tests showed that this window length is sufficiently long to distinguish between less polarized noise and more stable polarized signals. The minimum DOP threshold is set to 0.9 , that is we only use polarized signals with DOP $>0.9$ in the analysis. We then compute the median and the 16th and 84th percentiles of the distributions of the ellipticity measurements at each frequency and we compare the results to the theoretical ellipticity curves for fundamental mode and three overtones, calculated for the PREM model (Dziewonski \& Anderson 1981) using a normal mode approach (Gilbert 1970; Herrmann 2013). Results are plotted in Fig. 3. Here we can notice that the observed measurements (black dots with error bars) fit the theoretical value well for the fundamental mode in the period range $4.5-18 \mathrm{~s}$. The fit is not perfect at shorter periods but the theoretical value is still inside the measurement error bars. This is likely due to a combination of Love wave contamination and white uncorrelated noise, which cover the Rayleigh wave signals that are weaker at these periods. If we compare these results with those without Love wave contamination (Fig. $\mathrm{S} 3$ in the supplementary material) we notice a slightly improved fit for the higher frequencies, but still not perfect. In Fig. 3 errors are in general quite small but they increase when the ellipticity of the overtones is similar to the value of the fundamental mode. This is not surprising, because in this case the measurement of fundamental mode ellipticity is contaminated by overtones more strongly. We notice here that the ellipticity curve measured from DOP-E is
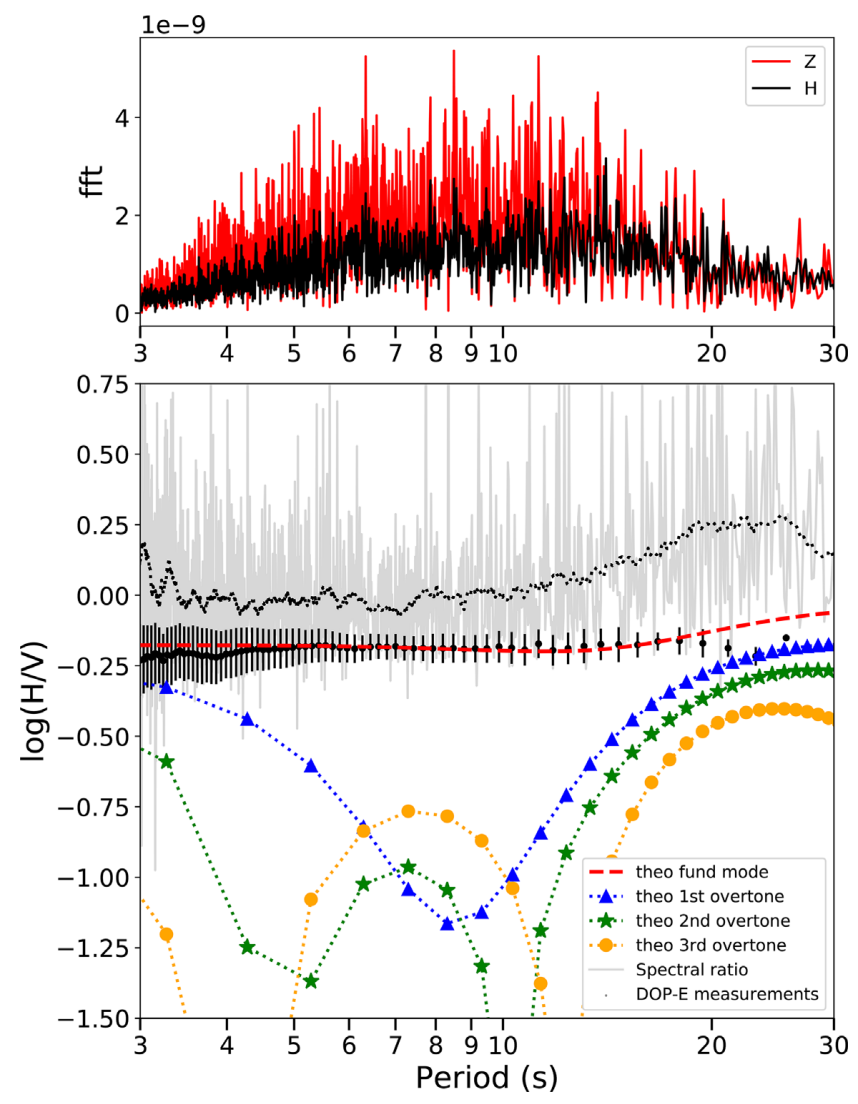

Figure 3. Top panel: spectra of three-component synthetic ambient noise simulated by modal summation using the PREM model (Dziewonski \& Anderson 1981), where $Z$ is the vertical component and $H$ is the horizontal component, defined as the root mean square of North and East components; bottom panel: Rayleigh wave ellipticity measured by DOP-E analysis applied to the synthetic ambient noise time series (black dots with error bars) compared to theoretical ellipticity computed for the PREM model (fundamental mode and three first overtones) and spectral ratio between horizontal and vertical components (dotted black line).

in general stable even with the contamination of noise with Love waves. On the other hand, the simple spectral ratio between horizontal and vertical components shows a general overestimation of ellipticity (dotted line in Fig. 3) due to the presence of Love waves in 


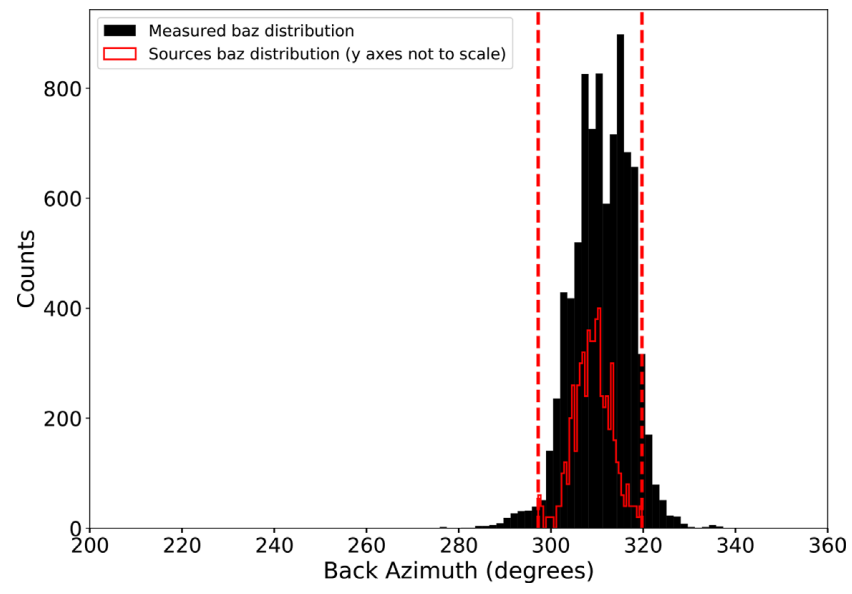

Figure 4. Histogram of backazimuth measured on synthetic ambient noise by DOP analysis (black) in the period band $4.5-15 \mathrm{~s}$. The true backazimuth distribution from the station PRMA to the sources is plotted in red (histogram not to scale) and it ranges between $299^{\circ}$ and $319^{\circ}$ (red dashed lines). Backazimuth is defined clock-wise from North ranging from $0^{\circ}$ to $360^{\circ}$.

the horizontal components. Since in real cases we do not know the location of the sources, we conservatively define here the horizontal component as the root mean square of North and East components, as suggested by Nakamura (1989). For comparison, Fig. S3 shows the same test adding white noise only, without the contamination of Love waves. Here both the spectral ratio and the DOP-E produce ellipticity curves that roughly match with the theoretical values. This is not surprising since the white noise equally affects both the vertical and horizontal components, so it does not affect the spectral ratio.

The DOP-E analysis is also able to retrieve the backazimuth of the polarized incoming signal. This capability can be useful for the characterization of the ambient noise sources, as shown by Schimmel et al. (2011). Here we plot the detected backazimuths (Fig. 4) compared with the real distribution of backazimuths from the sources to the receiver station (red histogram, not to scale). As we can note there is a good fit between the observed and the true backazimuth.

\section{SENSITIVITY ESTIMATE}

Synthetic tests including noise showed a good fit with theoretical values calculated on a known 1-D model. Rayleigh wave ellipticity has a very strong sensitivity to $v_{S}$ at shallow depth as shown, for example by the sensitivity kernels in Tanimoto \& Rivera (2008); sensitivity to shallower depth increases as the periods decreases. This means that Rayleigh wave ellipticity at a period of $\approx 2 \mathrm{~s}$ is sensitive to very shallow depth (in the order of hundreds of metres) . To test the resolution of Rayleigh wave ellipticity measurements to shallow $v_{S}$ variations we perform an additional synthetic test (Fig. 5). We choose a reference 1-D model (model 1, black line in panel a) and we perturb the top $500 \mathrm{~m}$ decreasing $v_{S}$ by 15 per cent (model 2 , red line in panel a). We then compute the theoretical ellipticity curves for both the models (panel b); the theoretical curves differ from each other by a maximum of 32 percent. We then simulate ambient noise using these two models and the same approach as shown in Section 3.1. Subsequently, we measure ellipticity using the DOP-E method on synthetic ambient noise simulated for the two subsurface models. Measurement results are shown in panel d. Here we see that, even if the error bars are slightly overlapping at the (a)
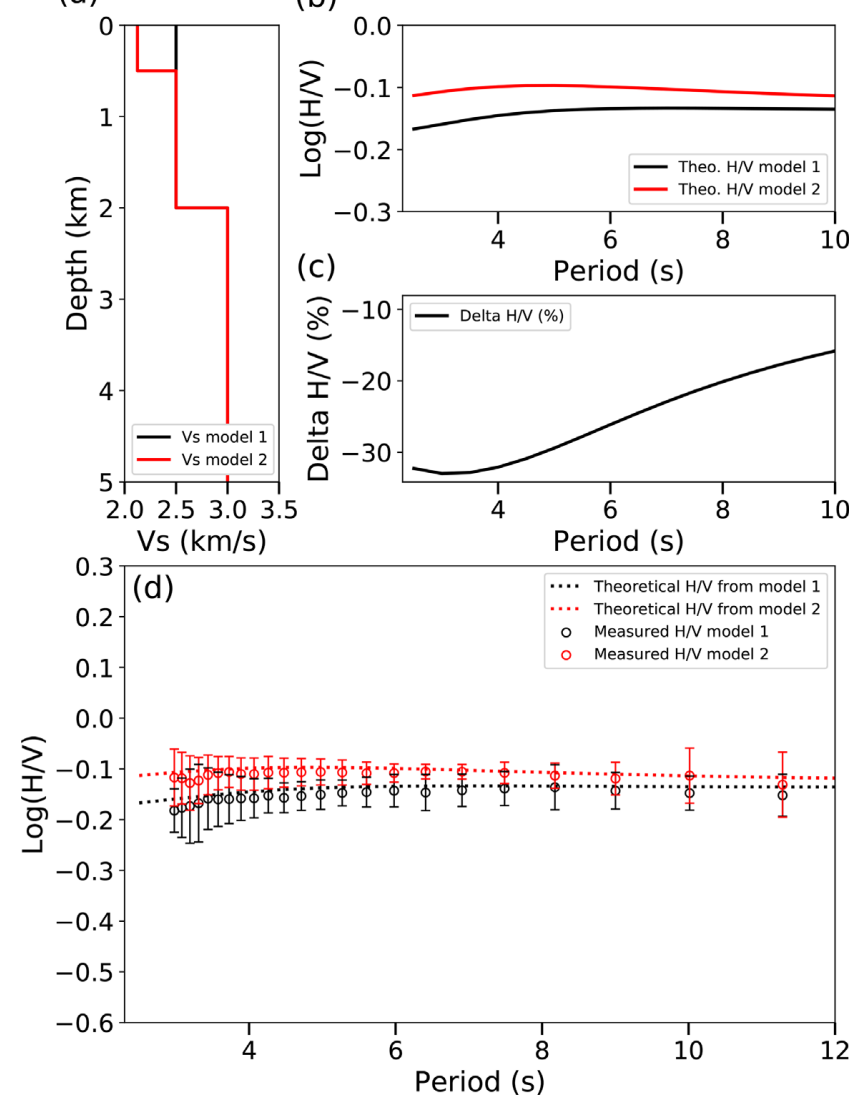

Figure 5. (a) Two shear wave velocity $\left(v_{S}\right) 1-\mathrm{D}$ depth profiles tested. The profiles are identical except in the top $500 \mathrm{~m}$, where $v_{S}$ in model 2 is 15 per cent larger than in model 1.; (b) Theoretical Rayleigh wave ellipticity calculated from model 1 (black) and model 2 (red); (c) percentual difference between theoretical ellipticity curves for models 1 and 2; (d) ellipticity measured by DOP-E analysis from synthetic ambient noise from model 1 (black dots with error bars) and model 2 (red dots with error bars) compared to the corresponding theoretical curves (black and red dotted lines).

shortest periods, it is possible to distinguish the two ellipticity curves and they fit well to the theoretical ones. This test demonstrates that using the DOP-E analysis on ambient noise it is possible to detect a variation of $v_{S}$ of 15 per cent in the top $500 \mathrm{~m}$ beneath the surface.

\section{INVERSION}

\subsection{Synthetic test}

In the previous section we demonstrated that using DOP-E analysis on ambient noise greatly enhances the sensitivity of ellipticity measurements to small structure variations at very shallow depth compared to using only measurements from distant earthquake data. Rayleigh wave ellipticity measurements on short-period data from distant earthquakes (teleseismic distances) are difficult because of attenuation reducing the signal-to-noise ratio. Thus, so far Rayleigh wave ellipticity studies based on distant earthquake data have been focused on wave periods of $T \approx 10 \mathrm{~s}$ or longer (e.g. Tanimoto \& Rivera 2008; Berbellini et al. 2017). In contrast, ambient noise measurements are stable at shorter wave periods $(T \approx 1-15 \mathrm{~s}$ in this study). By enabling the use of such short wave periods, ambient noise measurements image smaller scale structures and thus increase the resolution at shallow depths. In order to better quantify 
the capability of our measurement technique, we perform a synthetic inversion test. We choose an input $v_{S}$ model (red dashed line in left panels of Fig. 6) and we compute the theoretical ellipticity curve at the typical periods of ambient noise $(2-10 \mathrm{~s})$ and earthquake data (10-100 s). In order to simulate a realistic observation we add noise to the measurements: we compute 200 random curves from a Gaussian distribution with mean equal to the theoretical value for the PREM model and with standard deviation equal to that obtained from real measurements for station PRMA, which will be presented in Section 6.2.1. We finally compute for each wave period the mean of the 200 random points. This final ellipticity curve is used as input synthetic data in our inversions.

\subsection{Inversion technique}

We invert the ellipticity curve using the same approach as in Berbellini et al. (2017) and in Attanayake et al. (2017): they inverted ellipticity curves from teleseisms using the Neighbourhood Algorithm (Sambridge 1999). This self-adaptive method samples the model space in order to find an ensemble of models that best fit the real data. For each model sampled the inversion algorithm compares predicted to real data (we use the normal mode approach by Herrmann (2013) to compute ellipticity curves and evaluate a cost function). The cost function takes into account the misfit between real data and predicted values and it usually also contains a term to regularize the solutions. A regularization term is needed when the parametrization is made of many thin layers to foster the smoothest models. The cost function is defined as:

$c=\sum_{i=1}^{N_{m}} \frac{\left[d_{o b s}^{i}-g^{i}(m)\right]^{2}}{\left(\sigma_{D}^{i}\right)^{2}}+A \cdot N_{m} \sum_{j=1}^{N_{L}}\left[v_{S}^{j-1}-2 v_{S}^{j}+v_{S}^{j+1}\right]^{2}$,

where $d_{o b s}^{i}$ are the observed data, $g^{i}(m)$ are theoretical ellipticity values calculated using a normal mode formalism for the sampled model $m, \sigma_{D}^{i}$ is the variance of the measurement, $N_{m}$ is the number of measurements, $N_{L}$ is the number of layers, $A$ is a scaling factor, and $v_{S}$ is the shear-wave velocity. The first term is the misfit between observed and predicted data, the second is the regularization term. A is a scaling factor determined by trial-and-error: if A is too small, then the observed data will be over-fitted, while the resulting model will show very unrealistic discontinuities. On the other hand, if A is too large then the model will be flat and the observed data will not be fitted well. A good A factor produces realistic models while fitting well the observed data. In this test we remove the regularization in the cost function to put in evidence the resolution power of the measurement.

\subsection{Synthetic inversion test results}

Results of the synthetic inversion test are shown in Fig. 6. In the left panels we show the input model compared to the best model found. Also, in order to visualize the resolution of the models we plot in grey shadows all the models with a cost function within 20 per cent of the minimum cost function value obtained.

We first invert the ellipticity curve using only the periods from 10 to $100 \mathrm{~s}$ (Fig. 6B), corresponding to the frequency range of ellipticity measurements from earthquake records. In panel A, we show the best model found compared to the input one. We can notice that deep crustal structure is well constrained while the shallowest two layers are not well retrieved. We then invert the ellipticity curve using only the periods from 2 to $10 \mathrm{~s}$ (Fig. 6D), corresponding to the frequency band used in measurements from ambient noise.
Results (Fig. 6C) show that in this case the shallowest structure is well-constrained while deeper layers are not correct. The error bars of the ambient noise measurements in the period range $\approx 9-10 \mathrm{~s}$ are large because the signals are weaker in this period band (we recall here that error bars are taken from real measurements from station PRMA, that will be presented in Section 6.2.1). For shorter wave periods, the errors are in about the same order of magnitude as for the measurements based on earthquakes, apart from the period range 4-7 s, where they are lower because of the larger power of oceanic ambient noise in this period range. The large errors in the ambient noise measurements in the $9-10 \mathrm{~s}$ period range lead to larger uncertainties in the retrieved $v_{S}$ structure in the lower part of the shallow crust $(\approx 2-10 \mathrm{~km}$ depth, Fig. $6 \mathrm{C})$. We finally invert the whole curve from 2 to $100 \mathrm{~s}$ (Fig. 6F). In this case we find that the whole crustal structure is well-constrained (Fig. 6E). The $v_{S}$ errors in the lower part of the shallow crust $(\approx 2-10 \mathrm{~km})$ are smaller than when inverting the ambient noise measurements alone because the errors of the $T \approx 10 \mathrm{~s}$ measurements from earthquakes are lower than those from ambient noise. As expected the deepest layer is retrieved with lower resolution (i.e. with larger errors) than the shallower layers, which is similar to the findings of Berbellini et al. (2017).

\section{REAL DATA EXAMPLES}

\subsection{Concordia - Antarctica}

\subsubsection{Measurements}

We apply the DOP-E method described in Section 2.1 to real data to analyse the stability of the results and, in particular, to verify whether the Rayleigh wave ellipticity curve measured from ambient noise fits the ellipticity curve obtained from earthquake records by previous methods such as the one proposed by Berbellini et al. (2016) which is based on that of Tanimoto \& Rivera (2008). These methods measure ellipticity of Rayleigh waves by selecting time-windows in earthquake waveforms by the analysis of the phase-shift between horizontal (radial) and vertical components. As a first test we use data from the Concordia seismic station (CCD), a French-Italian permanent research station in the Antarctic plateau. We choose this station because of its very quiet location, without any local anthropic noise. Thus, the quality of records is very high, being ideally suited for a first test of the measurement scheme. We apply the DOP-E method to 1 month of records in January 2009. We apply the method using the measuring parameters presented in the supplementary material and described in Section 3.2.

Measurement results are shown in Fig. 7 where the black dots are the measurements from ambient noise by DOP-E in the period range $2-15 \mathrm{~s}$; red dots are measurements from earthquake signals using the method proposed by Berbellini et al. (2016). Here we used a data set of $\approx 600$ teleseisms from January 2009 to December 2013 with $M_{\mathrm{W}}>5.5$. We also show the theoretical ellipticity curve calculated from model CRUST1.0 (Laske et al. 2013) and LITHO1.0 (Pasyanos et al. 2014) using a normal mode approach. Fig. 7 shows that the curves obtained from the two methods overlap very well in the period range 10-15 s. Error bars are in general larger for ambient noise measurements at short periods. The comparison with the theoretical curve shows a good fit in the period band 10-60 s, while for shorter periods we notice larger discrepancies, reaching a maximum at about $4 \mathrm{~s}$. This suggests that the upper crust structure in the CRUST1.0 model near the CCD station is inaccurate. Thus, 

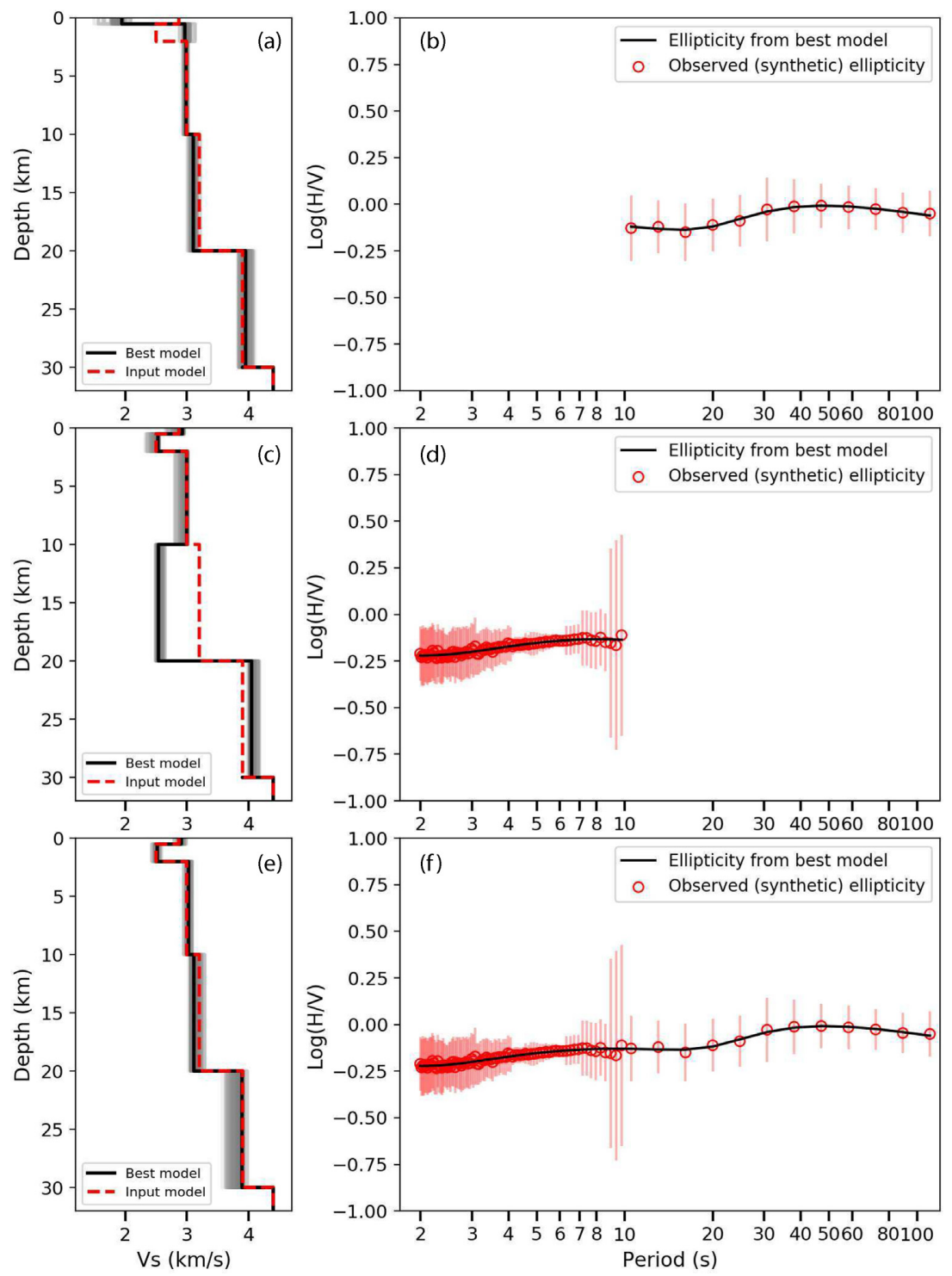

Figure 6. Synthetic test of the inversion scheme using earthquake periods only (top panels), using ambient noise periods only (central panels) and using the whole period range (bottom panels). Left-hand panels: input $v_{S}$ model (red dashed line); best model found (black line); models with cost function within 20 per cent from the best model (shadows of grey). Right-hand panels: synthetic curve computed from the input model with measurement noise added (red dots). Error bars are from real data measurements at station PRMA from earthquake data (panel B) and ambient noise data (panel D). Theoretical ellipticity curve from the best model found (black line).

the ellipticity measurements in the period range of 2-60 s can potentially help to better constrain the subsurface structure beneath the CCD station, notably in the upper crust.

In Fig. 8 we show the histogram of the backazimuth measured by DOP-E. The backazimuths have been determined from the orientation of the plane of motion, assuming that the particle motion for fundamental mode Rayleigh waves is retrograde at the surface, which is usually the case. From Fig. 8 it is evident that most of the Rayleigh waves arrive with a backazimuth of about $20^{\circ}$. In general an even azimuthal coverage would help to stabilize the ellipticity measurements, especially in the presence of strong lateral heterogeneity, given the sensitivity kernels shown by Maupin (2017). In this particular case we did not apply azimuthal normalization to clearly point out the capability of the DOP-E method to locate the direction of the ambient noise source. Moreover, we do not expect strong lateral heterogeneities in this particular geological setting.
The presence of a clear peak at $20^{\circ}$ shows that the ambient noise measured here has been possibly generated by a storm south of Cape of Good Hope, as is shown in Fig. 9(b). This region is often hit by a number of storms during the year, such as the one recorded in 2009 January January 10-12, that is the most likely source of the ambient noise recorded. This storm is well identified by the strong lowpressure anomaly in Fig. 9(b) (NOAA/ESRL Physical Sciences Division, Boulder Colorado http://www.esrl.noaa.gov/psd/). Another indication that the detected noise is generated by oceanic storms is given by the histogram of the backazimuth separated by period bands (Fig. S6). Here we see that the highest peak at $\approx 20^{\circ}$ is in the period band of the microseisms (3-10 s). Further DOP-E analysis from other stations in the area could potentially confirm and locate by triangulation the exact source of the seismic noise but such an analysis is beyond the purpose of this work. 


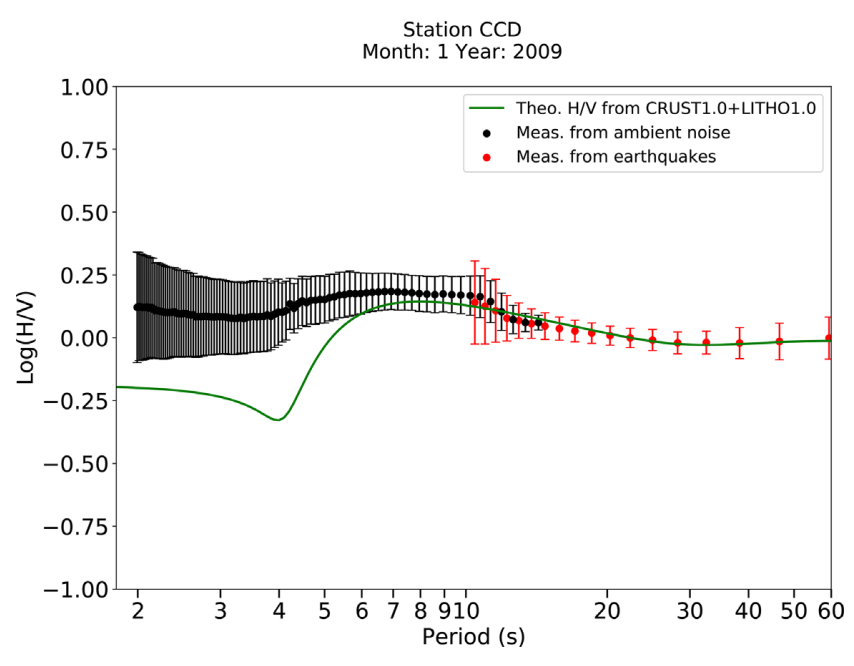

Figure 7. Black dots with error bars: ellipticity measurements on real ambient noise from Concordia station (Antarctica) using the DOP-E analysis. We used 1 month of records in January 2009. Red dots with error bars: ellipticity at the same station measured from earthquake data using the measurement technique by Berbellini et al. 2016. Green line: theoretical ellipticity calculated using a normal mode formalism for model CRUST1.0 (Laske et al. 2013) and LITHO1.0 (Pasyanos et al. 2014) at the location of the Concordia station.

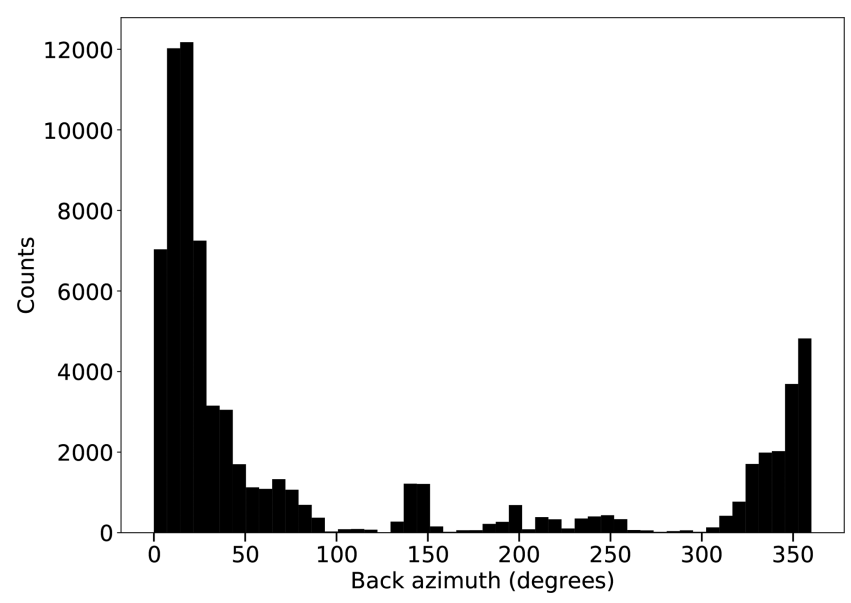

Figure 8. Histogram of backazimuth measured by DOP-E analysis on real ambient noise data at station Concordia for the month of January 2009.

\subsubsection{Inversion}

We then invert the Concordia ellipticity measurements shown in the previous section using the method described in Section 5.2. In this case the absence of a dense seismic network and strong seismicity can make an earthquake-based tomography of the crustal structure very difficult to perform. This is a perfect location to test the power of Rayleigh wave ellipticity measurements to retrieve the $v_{S}$ structure with few data and a single station. We paramtrize the structure beneath the station using six layers: two for the ice, three for the rocky crust and a layer between ice and rocks. This thin layer can potentially contain liquid water at a temperature of $\approx-2^{\circ}$ as demonstrated by many previous works (i.e. Tikku et al. 2005; Cianfarra et al. 2009; Wittlinger \& Farra 2015). We keep depth of the bedrock fixed at a depth of $\approx 3.1 \mathrm{~km}$, a value given by direct measurements from ice drilling (Rémy \& Tabacco 2000) but we leave the thickness of the three top layers free to vary during the inversion. We keep $v_{P}$ and $\rho$ in the ice fixed to the values measured by
Wittlinger \& Farra (2015) $\left(v_{P}=3.94 \mathrm{~km} \mathrm{~s}^{-1}\right.$ and $\left.\rho=0.94 \mathrm{~g} \mathrm{~cm}^{-3}\right)$, while for the third layer we set the density equal to 1 , to take into account the possibility that it contains liquid water. We fix the thickness of the three bottom rock layers to the values from LITHO1.0 (Pasyanos et al. 2014) and we scale $v_{P}$ and $\rho$ from $v_{S}$ using the Brocher relations (Brocher 2005). We do not apply any regularization here, since the model is relatively simple.

\subsubsection{Results}

We run the inversion sampling a total of 9000 models. Results are shown in Fig. 10. As we can see in the bottom panels, $v_{S}$ values for the rock part of the crust match very well with the ones from LITHO1.0. Resolution decreases slightly as depth increases due to the reduction of the sensitivity with depth. The shallowest thin layer found could potentially correspond to the firn layer that is usually estimated $\approx 150 \mathrm{~m}$ thick (King \& Jarvis 2007). This layer is composed by compacted snow and it is characterized by low shear waves velocities. In the rest of the ice layer the $v_{S}$ found is equal to $2.33 \mathrm{~km} \mathrm{~s}^{-1}$ and the average $v_{S}$ found in the glacier is $2.15 \mathrm{~km}$ $\mathrm{s}^{-1}$. This value differs slightly from the average value measured for the ice from receiver functions by Wittlinger \& Farra (2015) that is $v_{S}=1.81 \mathrm{~km} \mathrm{~s}^{-1}$. One of the most interesting features in the resulting model is the third layer: the thickness of this layer is extremely small, almost equal to 0 , meaning that the inversion tends to cancel out this layer. This result suggests that no liquid water is present beneath the ice at this location, nor low-velocity sediments at the base of the ice. This result is particularly interesting since the region of Concordia station is characterized by a number of subglacial lakes (Cianfarra et al. 2009), but no lake evidence has been found directly beneath the station. An interesting test would be to measure and invert ellipticity curves from seismic stations located above a well-known subglacial lake to verify if this method is able to locate it. Nevertheless this is well beyond the scope of this paper. We perform instead a preliminary synthetic test to empirically measure the sensitivity of ellipticity to variations of $v_{S}$ and thickness of a thin layer at the depth of an eventual lake. We use the best model found as a reference model but we vary $v_{S}$ of the 400-m-thick layer at the base of the ice and we compute the theoretical ellipticity curve for each model using a normal mode formalism. Results are shown in Fig. S9, which also shows as a reference the theoretical curve from the best model found with the standard deviation from real measurements at station Concordia. We observe that variations in $v_{S}$ produce a significant variation of the synthetic ellipticity curve, so the sensitivity of the measurement should be sufficient to locate low-velocity anomalies at this depth. We then do the same test assuming that the layer is made of liquid water $\left(v_{S}=0 \mathrm{~km} \mathrm{~s}^{-1}\right)$, we vary its thickness and we calculate the theoretical ellipticity curve for each model. Even in this case the predicted ellipticity curves show important differences, which highlights their sensitivity to such thin low velocity layers. Thus, if there is a low-velocity (or even liquid) layer at the base of the ice with a significant thickness ( $\gtrsim 100 \mathrm{~m}$ ), then the inversion of ellipticity measurements should be able to retrieve it.

\subsection{Parma - Italy}

\subsubsection{Measurements}

We then test the DOP-E method on data from a seismic station in a different geological setting. We choose station PRMA (Parma, Italy) 


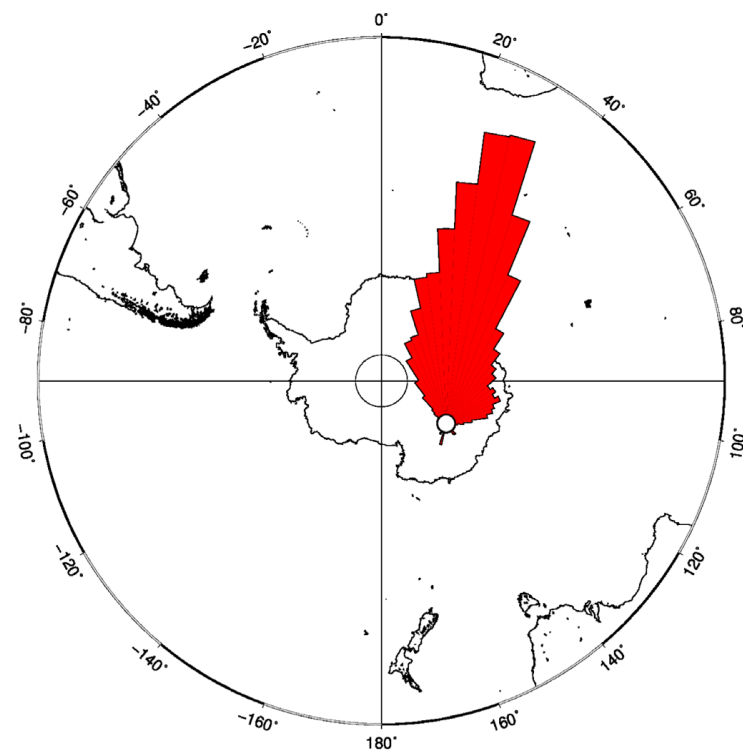

(a)

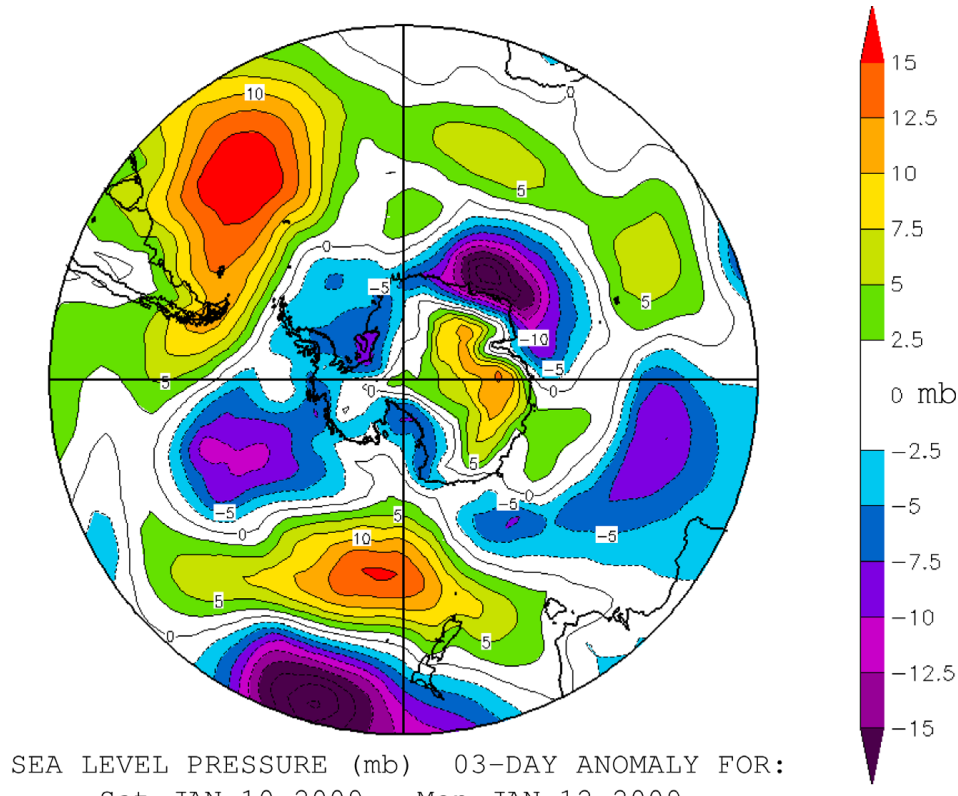

Sat JAN 102009 - MON JAN 122009

NCEP OPERATIONAL DATASET

(b)

Figure 9. Left-hand panel: 2-D histogram showing the geographical distribution of backazimuth measured at Concordia station. Right-hand panel: Air pressure anomaly at the surface recorded in the period 2009 January 10-12 by the NOAA/ESRL Physical Sciences Division (http://www.esrl.noaa.gov/psd/). The strong low-pressure anomaly in Northern-East Antartica coast is a good indicator of an oceanic storm hitting the coast and lasting approximately $3 \mathrm{~d}$.

located on a well-known sedimentary basin in Northern Italy, the Po Plain. We use one month of data in June 2010. In this case we expect a much worse quality of data compared to the CCD station because of local disturbances from anthropic activities and the different geological setting: since the station is located on a sedimentary basin, near its borders, we expect that the signal is more affected by local scattering and focusing-defocussing (Stich et al. 2009). As in the previous case we use the measurement parameters shown in the supplementary material.

Results of the measurements are shown in Fig. 11. Here we compare measurements from ambient noise (black dots) with measurements from earthquake (Berbellini et al. 2016, red dots) and theoretical ellipticity values calculated using the region's earth models MAMBo (Molinari et al. 2015, green line) and MAMBo-E (Berbellini et al. 2017, blue line). We can notice that the ellipticity curves from the two measurement methods fit quite well with each other in the period range $10-15 \mathrm{~s}$ but the errors in the ambient noise measurements are much larger than in the previous case (CCD station). This is possibly due to the different frequency content in the noise recorded by the two stations, as shown in Fig. S8. Here we notice that PRMA records contain more low-period signals (), possibly due to anthropic activities, and less oceanic noise around the wave periods of $6-9 \mathrm{~s}$. Larger errors in the period band 6-9s are then possibly due to the lower power of oceanic noise. Error bars decrease for shorter periods with minimum values in the period range $4-6 \mathrm{~s}$. This is quite surprising, since the power spectra showed in Fig. S8 does not show particularly large signals at this period band. Such lower errors are then possibly due to ambient noise that is more polarized at these wave periods, but the precise reason for this behaviour is still an open question. Also, the very complex geological setting beneath PRMA station, laying on the borders of a large and thick sedimentary basin, makes the wave propagation quite complex, due to internal reverberations, as well as focussing and defocussing effects. Hence it is not surprising that the results for the PRMA station show larger errors and are more complex to interpret than for CCD station. The ellipticity measurements fit quite well with the theoretical curve from MAMBo-E, while the differences to the theoretical curves computed for MAMBo are quite large. This is not surprising since MAMBo-E is a crustal model obtained from the update of MAMBo using ellipticity measurements from earthquake data (see Berbellini et al. 2017). In Fig. 12 we also show the backazimuth measured using the DOP-E analysis. In this case the location of the ambient noise sources is not clear (as shown also in Fig. S7) and further analysis would be needed to better locate it.

\subsubsection{Inversion}

We invert the whole ellipticity curve using the technique described in Section 5.2. We choose a parametrization with 16 layers, with fixed thickness ranging between $0.1 \mathrm{~km}$ at the surface and $9 \mathrm{~km}$ at the bottom of the crust. $v_{S}$ in each layer is constant and $v_{P}$ and $\rho$ are scaled from $v_{S}$ using the Brocher relations (Brocher 2005). Since the layers are very thin we apply here a regularization to avoid very oscillatory and unrealistic solutions that can fit the data well. We then apply the second term in the cost function (eq. 1) with $A=$ $10^{-2}$. This value has been determined by trial-and-error.

\subsubsection{Results}

We run the inversion sampling a total of 42000 models. Results are shown in Fig. 13. In the bottom panels we show the best-fitting model found (red line) compared to the model MAMBo-E, which has been determined by Berbellini et al. (2016) from the inversion of ellipticity measurements from earthquake records only. The grey lines correspond to models with a cost function within 20 per cent 

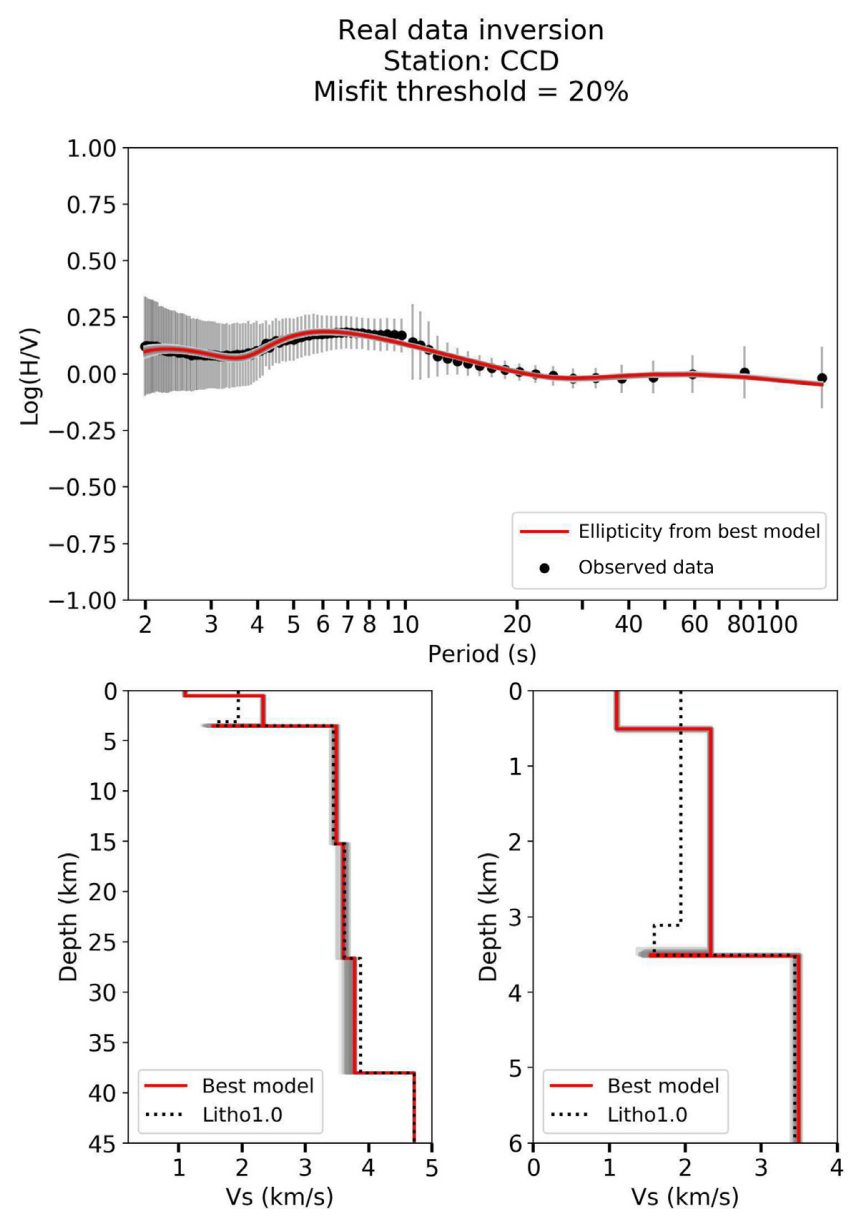

Figure 10. Inversion results from real data measurements at station CCD (Concordia station, Antarctica) from both ambient and earthquakes. Top panel: Observed data (black dots with error bars); ellipticity computed from the best value found (red line); ellipticity computed from models with cost function within 20 per cent from the best model (shadows of grey). Bottom panels: Best model found (red solid line); models with cost function within 20 percent from the best model (shadows of grey); model LITHO1.0 by Pasyanos et al. (2014) (black dashed line). Bottom right-hand panel: Zoom of the shallowest $6 \mathrm{~km}$.

of the best-fitting model, which give an empirical estimate of the errors. We can notice an overall match between the final model found and the reference model MAMBo-E with some interesting differences: the final model has a quite large discontinuity at $\approx 5 \mathrm{~km}$ depth that is not present in MAMBo-E. Also, $v_{S}$ is larger than the reference model in the depth range $0.5-1.5 \mathrm{~km}$. It is interesting to notice that the top, thin layer is well constrained, confirming the value found in MAMBo-E. In the top panel is shown the best predicted ellipticity curve compared with the observed one. It is worth to remember that the reference model MAMBo-E is not used in the inversion but plotted here only for comparison. This test shows that the inversion of the ellipticity curve in the whole period band from 2 to $100 \mathrm{~s}$ retrieves a $v_{S}$ structure beneath the receiver station that is compatible with previous studies but adding new details, especially at shallow depth. This result demonstrates that by using a wider period band than before it is possible to constrain a reliable model even if the thickness and depth of the layers is not imposed a priori, as it has been done for MAMBo-E. For comparison, we perform an inversion using exactly the same technique but with only data from earthquakes (in the period range 10-100 s). Results (in

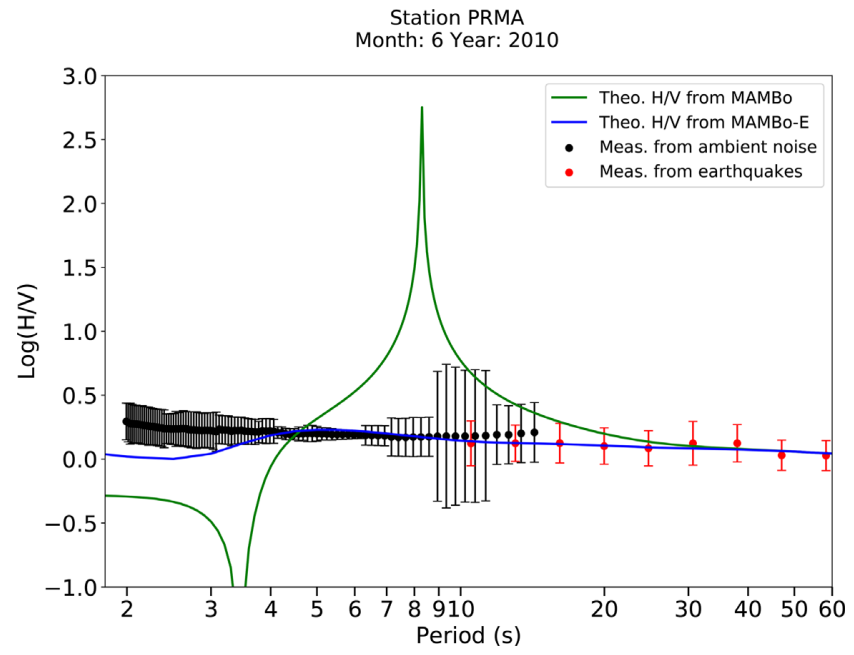

Figure 11. Black dots with error bars: Rayleigh wave ellipticity measurements on real ambient noise from PRMA station (Parma, Italy) using the DOP-E analysis. We used one month of records in June 2010. Red dots with error bars: ellipticity at the same station measured from earthquake data from Berbellini et al. (2016). Green line: theoretical ellipticity calculated using a normal mode formalism for model MAMBo (Molinari et al. 2015). Blue line: theoretical ellipticity calculated using a normal mode formalism for model MAMBo-E (Berbellini et al. 2017) at the location of the Parma station.

Fig. S10) show that in this case such a parametrization with thin layers produces very unstable models and unrealistic values.

\section{DISCUSSION AND FUTURE APPLICATIONS}

The synthetic test presented in Section 5 and the real data inversion tests showed that using ellipticity measurements in a broad frequency band (2-100 s) improves significantly the capability to retrieve the whole $v_{S}$ crustal structure from the shallowest to the deepest parts of the crust compared to the use of smaller frequency bands. Inverting single-station ellipticity measurements from ambient noise only is possible but its sensitivity will be limited to the top $\approx 10 \mathrm{~km}$. Moreover, the retrieved structure for the lower part of the upper crust may show larger errors than when jointly inverting measurements from earthquakes and ambient noise. The use of ambient noise recorded at a single station instead of earthquake records (or, perhaps instead of noise cross-correlation between pairs of stations) allows to perform ellipticity measurements with relatively short time windows: while measurements from earthquake data typically require more than 150 teleseismic events (usually $\approx 2$ yr of data), DOP-E analysis of ambient noise can be performed with only 1 month of data or less, as performed in this study. This feature, together with the strong sensitivity of ellipticity to small variations of $v_{S}$ at shallow depth, could make DOP-E an exciting tool to investigate a wide range of problems, for example in volcanoes, fault areas, glaciers, etc. For example, transients and trends of $v_{S}$ in time, which are already being investigated with interstation ambient noise analysis (e.g. Brenguier et al. 2008; Duputel et al. 2009 on the Reunion volcano, Schaff 2012 on the Parkfield earthquake, Minato et al. 2012 on the 2011 Tohoku-oki earthquake and Zaccarelli et al. 2011 on the 2009 L'Aquila earthquake), could be potentially examined. The detailed scope for such applications needs to be thoroughly studied in future work. Furthermore, the relatively small amount of data needed for a DOP-E analysis on 

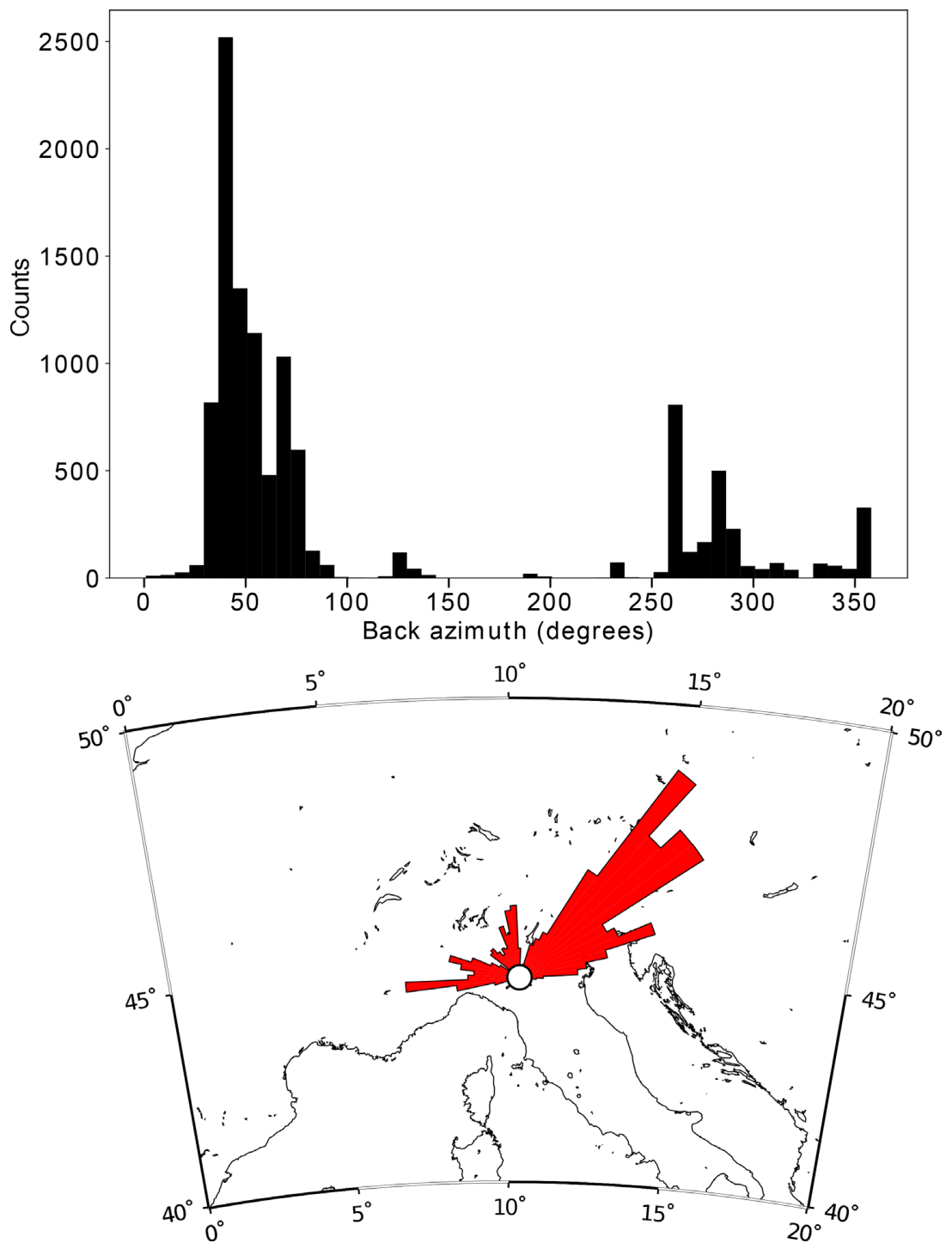

Figure 12. Top panel: Histogram of backazimuth measured by DOP-E analysis on real ambient noise data at station PRMA for the month of June 2010. Bottom panel: 2-D histogram showing the geographical distribution of backazimuth measured at PRMA station.

ambient noise makes it a useful tool for exploring data from short temporary seismic deployments. Another important feature of the DOP-E analysis is that it also gives information on the backazimuth of the ambient noise recorded. This allows a characterization of the seismic noise sources that is not possible with traditional singlestation H/V methods, such as by Nakamura (1989). Moreover, since it is a single-station technique, it can be used when a dense seismic array is not available. In this sense it could be an alternative to the recent methods proposed for instance by Hobiger et al. (2016) and Maranò et al. (2017). These recent methods provide very accurate measurements of Rayleigh wave ellipticity for fundamental mode and overtones, polarization and backazimuth, but since they use dense arrays they cannot always be used in seismological applications. Also, they have been developed and tested primarily to study the top hundreds of meters in the subsurface, whereas the DOP-E method enables the imaging of the top kilometres of the subsurface structure from ambient noise, and of the whole crust when combining ambient noise and earthquake data. The DOP-E technique showed a good capability of separating Love wave from Rayleigh wave noise, as shown in the synthetic validation test. $\mathrm{H} / \mathrm{V}$ spectral ratios (Nakamura 1989), currently the most-widely used method to retrieve Rayleigh-wave ellipticity from noise records, cannot prevent contamination from Love waves, hence they often overestimate the ratio. Ellipse-fitting methods, either based on single three-component stations (e.g. DELFI, Hobiger \& Bihan 2009; RayDec, Hobiger et al. 2009) or on arrays of stations (e.g. $f-k$ analysis, Poggi et al. 2012; MUSIQUE, Hobiger et al. 2016; WaveDec, Maranò et al. 2017) reduce, or eliminate, such contamination and provide more reliable estimates of ellipticity identifying the azimuth of incidence. Array methods may also break the intrinsic $180^{\circ}$ ambiguity, thus allowing determination of prograde versus retrograde particle motion. Such methods have primarily targeted a period range roughly between 0.1 and $1 \mathrm{~s}$-and the top $\approx 300 \mathrm{~m}$ of earth structure. DOP-E also identifies polarization parameters of Rayleigh wave motion, and we show here examples of application in the period band from 1 to $10 \mathrm{~s}$, to obtain continuity with measurements on earthquake data and constrain $v_{S}$ structure in the crust. An extensive comparison among these methods is beyond the scope of this study, and their performance may vary in different applications. We are providing the software tools that implement DOP-E (see 

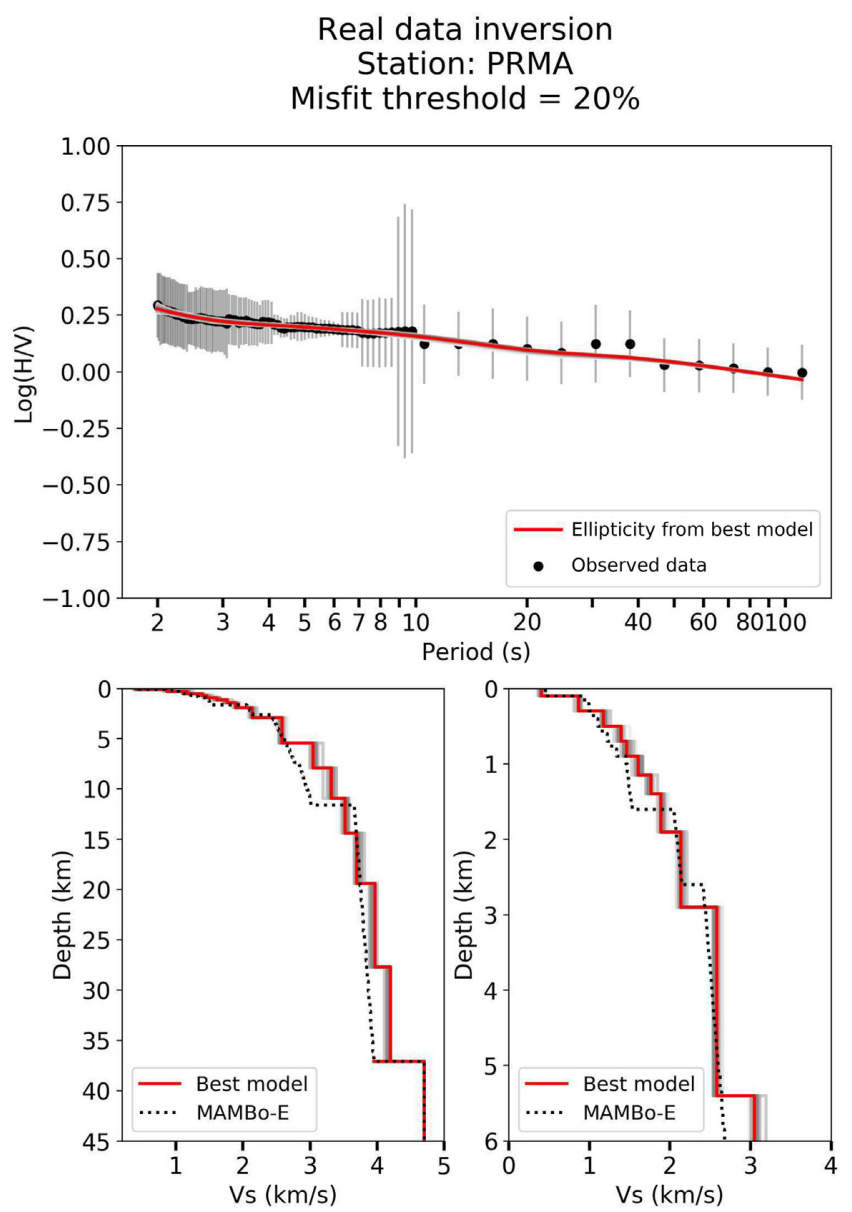

Figure 13. Inversion results from real data measurements at station PRMA (Parma, Italy) from both ambient and earthquakes. Top panel: Observed data (black dots with error bars); ellipticity computed from the best value found (red line); ellipticity computed from models with cost function within 20 per cent from the best model. Bottom panels: Best model found (red solid line); models with cost function within 20 per cent from the best model (shadows of grey); model MAMBo-E by Berbellini et al. (2017) (black dashed line). Bottom right-hand panel: Zoom of the shallowest $6 \mathrm{~km}$.

Section Data and software below). The DOP-E technique presented in this study may be particularly useful for regions with low levels of local or regional seismicity. On the other hand, in seismic active areas (e.g. California, Japan) the DOP-E method can be used also to measure short-period ellipticity from local earthquakes and to constrain the shallow structure. In fact we do not need to consider how the Rayleigh waves have been generated. We ideally just want to use a broad frequency band to enhance the inversion resolution. Since at a given frequency and azimuth the Rayleigh wave ellipticity should depend only on the local Earth structure, Rayleigh wave recordings from both earthquakes and noise can be analysed in one single run without knowing their sources. Further possible research directions include using measurements from different stations to locate the sources of noise at different periods and to possibly distinguish between anthropogenic and natural sources (e.g. storms and oceanic waves). Moreover, as an expansion of this work, in the future it may be possible to perform more precise measurements applying an azimuthal normalization to obtain an even coverage of the sources.

Finally, other future research could include synthetic tests considering 3-D lateral variations in the background models, but these are well beyond the scope of this study as they would involve the computation of relatively high-frequency waveforms for laterally varying earth models, which are computationally very challenging. However, we may point out that Maupin (2017) showed that 3-D sensitivity kernels of Rayleigh wave ellipticity can exhibit substantial complexity. For example, the kernels with respect to $v_{S}$ show alternating positive and negative values both in depth and in distance from receiver. Thus, care needs to be taken when interpreting velocity models based on Rayleigh wave ellipticity data; future synthetic tests considering 3-D earth models may indeed help guide the interpretation.

\section{CONCLUSIONS}

The new method DOP-E showed a good capability of detecting Rayleigh waves from ambient noise records and measuring Rayleigh wave ellipticity using a single-station technique. The synthetic validation tests showed a good fit with theoretical values from a 1-D model. Real data examples from stations CCD and PRMA demonstrate that new ellipticity measurements from ambient noise are consistent with those from earthquake data for the wave periods for which it is possible to perform the two types of measurements. The very strong sensitivity of ellipticity to shallow and small variations of $v_{S}$, together with the short time window records needed to perform a measurement, make this method a potentially useful tool to analyse transients in $v_{S}$ values. This could be useful to study the time dependent $v_{S}$ trends in several areas, such as volcanic settings, fault areas before and after a big earthquake, ice melting, permafrost and so on. These applications deserve future investigations. Moreover, the backazimuth analysis that is performed by this method would also permit a better characterization of the ambient noise source. DOP-E could be a complementary alternative to the traditional Nakamura method and other more recent techniques, notably based on array data, for a variety of seismological applications.

It also allows to enlarge the frequency band of ellipticity measurements from teleseisms down to small periods. The use of measurements with such a wide frequency band enables the determination of the detailed structure of shear-wave velocity from the shallowest hundreds of meters down to the Moho, even when the depths of crustal discontinuities are not known, as we demonstrated for station PRMA. Results from the two inversions of real measurements presented here from Parma (Italy) and Concordia station (Antarctica) showed a good agreement with previous models. Particularly interesting is the case of Concordia station, where our results suggest that there is no liquid water at the base of the ice; we can envisage further applications of the method to other locations on the Antarctic platform.

\section{DATA AND SOFTWARE}

Seismic data from Parma seismic station were provided by IV seismic network (http://eida.rm.ingv.it/). Concordia seismic station is jointly operated by INGV (Istituto Nazionale di Geofisica e Vulcanologia, Rome) and GEOSCOPE (Institut De Physique Du Globe De Paris and Ecole Et Observatoire Des Sciences De La Terre De Strasbourg 1982). Concordia $\left(75^{\circ} \mathrm{S} 123^{\circ} \mathrm{E}\right)$ is a French-Italian Antarctic base funded by their polar institutes: IPEV (Institut Polaire Français) and PNRA (Programma Nazionale di Ricerche in Antartide, Italy). All seismic waveforms were collected from ORFEUSEIDA data centers. Source routines to perform DOP-E measurements and to invert Rayleigh wave ellipticity curves, together with 
technical documentation and examples can be downloaded from: github.com/berbellini/DOP-E.

\section{ACKNOWLEDGEMENTS}

This work received a fundamental contribution by TIDES COST Action ES1401 (in particular by Short Term Scientific Mission program). A.M.G. Ferreira and A. Berbellini are also grateful for funding from NERC project NE/N011791/1. M. Schimmel acknowledges funding by the Spanish MISTERIOS project (CGL201348601-C2-1-R). We also thank Lucia Gualtieri for the precious suggestions and discussions about ambient noise simulation.

We are very thankful to the anonymous referees for their careful and constructive reviews to improve our manuscript and Editor L. Boschi for all work involved with handling the manuscript.

\section{REFERENCES}

Attanayake, J., Ferreira, A.M., Berbellini, A. \& Morelli, A., 2017. Crustal structure beneath Portugal from teleseismic Rayleigh Wave Ellipticity, Tectonophysics, 712, 344-361.

Berbellini, A., Morelli, A. \& Ferreira, A.M., 2016. Ellipticity of Rayleigh waves in basin and hard-rock sites in Northern Italy, Geophys. J. Int., 206(1), 395-407.

Berbellini, A., Morelli, A. \& Ferreira, A. M.G., 2017. Crustal structure of Northern Italy from the ellipticity of Rayleigh Waves, Phys. Earth planet. Inter, 265, 1-14.

Brenguier, F., Shapiro, N.M., Campillo, M., Ferrazzini, V., Duputel, Z., Coutant, O. \& Nercessian, A.. 2008. Towards forecasting volcanic eruptions using seismic noise, Nat. Geosci., 1(2), 126-130.

Brocher, T.M., 2005. Empirical relations between elastic wavespeeds and density in the Earth's crust, Bull. seism. Soc. Am., 95, 2081-2092.

Cianfarra, P., Forieri, A., Salvini, F., Tabacco, I.E. \& Zirizotti, A., 2009. Geological setting of the Concordia Trench-Lake system in East Antarctica, Geophys. J. Int., 177(3), 1305-1314.

Duputel, Z., Ferrazzini, V., Brenguier, F., Shapiro, N., Campillo, M. \& Nercessian, A., 2009. Real time monitoring of relative velocity changes using ambient seismic noise at the Piton de la Fournaise volcano (La Réunion) from January 2006 to June 2007, J. Volc. Geotherm. Res., 184(1-2), 164 173

Dziewonski, A.M. \& Anderson, D.L., 1981. Preliminary reference Earth model, Phys. Earth planet. Inter., 25(4), 297-356.

Fäh, D., Kind, F. \& Giardini, D., 2003. Inversion of local S-wave velocity structures from average $\mathrm{H} / \mathrm{V}$ ratios, and their use for the estimation of site-effects, J. Seismol., 7(4), 449-467.

Ferreira, A.M. \& Woodhouse, J.H., 2007a. Observations of long period Rayleigh wave ellipticity, Geophys. J. Int., 169(1), 161-169.

Ferreira, A.M. \& Woodhouse, J.H., 2007b. Source, path and receiver effects on seismic surface waves, Geophys. J. Int., 168(1), 109-132.

Friedrich, A., Krueger, F. \& Klinge, K., 1998. Ocean-generated microseismic noise located with the Gräfenberg array, J. Seismol., 2(1), 47-64.

Gilbert, F., 1970. Excitation of the normal modes of the earth by earthquake sources, Geophys. J. R. astr. Soc., 22(2), 223-226.

Gualtieri, L., Stutzmann, E., Capdeville, Y., Ardhuin, F., Schimmel, M., Mangeney, A. \& Morelli, A., 2013. Modelling secondary microseismic noise by normal mode summation, Geophys. J. Int., 193(3), 1732-1745.

Haubrich, R.A., Munk, W.H. \& Snodgrass, F.E., 1963. Comparative spectra of microseisms and swell, Bull. seism. Soc. Am., 53(1), 27-37.

Herrmann, R.B., 2013. Computer Programs in Seismology: an Evolving Tool for Instruction and Research, Seismol. Res. Lett., 84(6), 1081-1088.

Hobiger, M. \& Bihan, N.L., 2009. Rayleigh wave ellipticity estimation from ambient seismic noise using single and multiple vector-sensor techniques, in Proceedings of the 17th Eur. Signal Process. Conf., pp. 2037-2041, Eusipco.
Hobiger, M., Bard, P.Y., Cornou, C. \& Le Bihan, N., 2009. Single station determination of Rayleigh wave ellipticity by using the random decrement technique (RayDec), Geophys. Res. Lett., 36(14), 1-5.

Hobiger, M. et al., 2013. Ground structure imaging by inversions of Rayleigh wave ellipticity: sensitivity analysis and application to European strongmotion sites, Geophys. J. Int., 192(1), 207-229.

Hobiger, M., Cornou, C., Bard, P.Y., Bihan, N.L. \& Imperatori, W., 2016. Analysis of seismic waves crossing the Santa Clara Valley using the three-component MUSIQUE array algorithm, Geophys. J. Int., 207(1), 439-456.

King, E.C. \& Jarvis, E.P., 2007. Use of shear waves to measure Poisson's ratio in polar firn, J. Environ. Eng. Geophys., 12(1), 15-21.

Laske, G., Masters, G., Ma, Z. \& Pasyanos, M., 2013. Update on CRUST1.0 - A 1-degree Global Model of Earth's Crust, Geophys. Res. Abstr., 15, EGU General Assembly 2013, held 7-12 April, 2013 in Vienna, Austria, id. EGU2013-2658.

Lin, F.C., Schmandt, B. \& Tsai, V.C., 2012. Joint inversion of Rayleigh wave phase velocity and ellipticity using USArray: constraining velocity and density structure in the upper crust, Geophys. Res. Lett., 39(12), doi:10.1029/2012GL052196.

Maranò, S., Hobiger, M. \& Fäh, D., 2017. Retrieval of Rayleigh wave ellipticity from ambient vibration recordings, Geophys. J. Int., 209(1), 334-352.

Maupin, V., 2017. 3-D sensitivity kernels of the Rayleigh wave ellipticity, Geophys. J. Int., 211(1), 107-119.

Minato, S., Tsuji, T., Ohmi, S. \& Matsuoka, T., 2012. Monitoring seismic velocity change caused by the 2011 Tohoku-oki earthquake using ambient noise records, Geophys. Res. Lett., 39(9), 1-6.

Molinari, I., Argnani, A., Morelli, A. \& Basini, P., 2015. Development and testing of a $3 \mathrm{D}$ seismic velocity model of the Po plain sedimentary Basin, Italy, Bull. seism. Soc. Am., 105(2A), 753-764.

Nakamura, Y., 1989. A method for dynamic characteristics estimation of subsurface using microtremor on the ground surface, Q. Rep. RTRI, 30(1), $25-33$.

Nishida, K., Kawakatsu, H., Fukao, Y. \& Obara, K., 2008. Background Love and Rayleigh waves simultaneously generated at the Pacific Ocean floors, Geophys. Res. Lett., 35(16).

Obrebski, M.J., Ardhuin, F., Stutzmann, E. \& Schimmel, M., 2012. How moderate sea states can generate loud seismic noise in the deep ocean, Geophys. Res. Lett., 39(11), 1-6.

Pastén, C., Sáez, M., Ruiz, S., Leyton, F., Salomón, J. \& Poli, P., 2016. Deep characterization of the Santiago Basin using HVSR and cross-correlation of ambient seismic noise, Eng. Geol., 201, 57-66.

Pasyanos, M.E., Masters, T.G., Laske, G. \& Ma, Z., 2014. LITHO1.0: An updated crust and lithospheric model of the Earth, J. Geophys. Res.: Solid Earth, 119(3), 2153-2173.

Poggi, V., Fäh, D., Burjanek, J. \& Giardini, D., 2012. The use of Rayleighwave ellipticity for site-specific hazard assessment and microzonation: application to the city of Lucerne, Switzerland, Geophys. J. Int., 188(3), $1154-1172$.

Rémy, F. \& Tabacco, I.E., 2000. Bedrock features and ice flow near the EPICA Ice Core Site (Dome C, Antarctica), Geophys. Res. Lett., 27, 405-408.

Sambridge, M., 1999. Geophysical inversion with a neighbourhood algorithm-I. Searching a parameter space, Geophys. J. Int., 138(2), 479494.

Sánchez-Sesma, F.J. et al., 2011. A theory for microtremor H/V spectral ratio: application for a layered medium, Geophys. J. Int., 186(1), 221225 .

Schaff, D.P., 2012. Placing an upper bound on preseismic velocity changes measured by ambient noise monitoring for the $2004 \mathrm{Mw} 6.0$ Parkfield Earthquake (California), Bull. seism. Soc. Am., 102(4), 1400.

Scherbaum, F.K.H. \& Ohrnberger, M., 2003. Determination of shallow shear wave velocity profiles in the Cologne, Germany area using ambient vibrations, Geophys. J. Int, 152(1984), 597-612.

Schimmel, M. \& Gallart, J., 2003. The use of instantaneous polarization attributes for seismic signal detection and image enhancement, Geophys. J. Int., 155(2), 653-668. 
Schimmel, M. \& Gallart, J., 2004. Degree of polarization filter for frequencydependent signal enhancement through noise suppression, Bull. seism. Soc. Am., 94(3), 1016-1035.

Schimmel, M., Stutzmann, E., Ardhuin, F. \& Gallart, J., 2011. Polarized Earth's ambient microseismic noise, Geochem., Geophys. Geosyst., 12(7), $1-14$.

Stich, D., Danecek, P., Morelli, A. \& Tromp, J., 2009. Imaging lateral heterogeneity in the Northern Apennines from time reversal of reflected surface waves, Geophys. J. Int., 177(2), 543-554.

Stockwell, R.G., Mansinha, L. \& Lowe, R.P., 1996. Localization of the complex spectrum: the S transform, IEEE Trans. Signal Process., 44(4), 998-1001.

Tanimoto, T. \& Rivera, L., 2008. The ZH ratio method for long-period seismic data: sensitivity kernels and observational techniques, Geophys. J. Int., 172(1), 187-198.

Tanimoto, T., Ishimaru, S. \& Alvizuri, C., 2006. Seasonality in particle motion of microseisms, Geophys. J. Int., 166(1), 253-266.

Tanimoto, T., Hadziioannou, C., Igel, H., Wasserman, J., Schreiber, U. \& Gebauer, A., 2015. Estimate of Rayleigh-to-Love wave ratio in the secondary microseism by colocated ring laser and seismograph, Geophys. Res. Lett., 42(8), 2650-2655.

Tikku, A.A., Bell, R.E., Studinger, M., Clarke, G.K., Tabacco, I. \& Ferraccioli, F., 2005. Influx of meltwater to subglacial Lake Concordia, East Antarctica, J. Glaciol., 51(172), 96-104.

Wittlinger, G. \& Farra, V., 2015. Evidence of unfrozen liquids and seismic anisotropy at the base of the polar ice sheets, Polar Sci., 9(1), 66-79.

Woodhouse, J.H., 1974. Surface waves in a laterally varying layered structure, Geophys. J. R. astr. Soc., 37(3), 461-490.

Yano, T., Tanimoto, T. \& Rivera, L., 2009. The ZH ratio method for longperiod seismic data: Inversion for S-wave velocity structure, Geophys. $J$. Int., 179(1), 413-424.

Zaccarelli, L., Shapiro, N.M., Faenza, L., Soldati, G. \& Michelini, A., 2011. Variations of crustal elastic properties during the 2009 L'Aquila earthquake inferred from cross-correlations of ambient seismic noise, Geophys. Res. Lett., 38(24).

\section{SUPPORTING INFORMATION}

Supplementary data are available at $G J I$ online.

Table S1. Parameter values used in the DOP-E measurement scheme.

Figure S1. (a): Comparison between time series and Fourier amplitude spectra of synthetic ambient noise containing Rayleigh wave only (red) and simulated ambient noise containing also white noise with a signal-to-noise ratio equal to 30 . (b): top panel: vertical (red) and horizontal (black) components of the simulated ambient noise; bottom panel: ellipticity curve measured from the simulated ambient noise using the DOP-E method (black dots), theoretical ellipticity computed from model Prem (Dziewonski \& Anderson
1981) (red dashed line), spectral ratio (grey line) and its running average (dotted black line) and ellipticity of the first 3 overtones (coloured dashed lines).

Figure S2. Same as Fig. S1 but using a signal-to-noise ratio equal to 10 .

Figure S3. Same as Fig. S1 but using a signal-to-noise ratio equal to 7.5 .

Figure S4. Same as Fig. S1 but using a signal-to-noise ratio equal to 5 .

Figure S5. Same as Fig. S1 but using a signal-to-noise ratio equal to 2 .

Figure S6. Histogram of the measured backazimuths for station Concordia (CCD) separated by period bands.

Figure S7. Histogram of the measured backazimuths for station Parma (PRMA) separated by period bands.

Figure S8. Power spectra from data from station CCD (black) and PRMA (red).

Figure S9. Sensitivity test for a thin layer at $\approx 3 \mathrm{~km}$ depth. Top panels: test of the sensitivity to $v_{S}$ variations. Left: Black line: best model found in the inversion (fig. 10 in the main text); black dotted line: LITHO1.0 model (Pasyanos et al. 2014); coloured lines: test models; Right: Black line with errorbars: ellipticity curves computed by modal summation from input model with standard deviations from real data measurements at station Concordia; coloured lines: ellipticity computed from corresponding models on the left panel. Bottom panels: test of the sensitivity to the thickness of a layer of liquid water at $\approx 3 \mathrm{~km}$ depth. Left: Black line: best model found; coloured lines: test models. Right: Black line with error bars: ellipticity computed from model LITHO1.0 and standard deviations from real data measurements from station Concordia; Coloured lines: ellipticity computed from corresponding models plotted in the left panel.

Figure S10. Inversion results from real data measurements at station PRMA (Parma, Italy) using earthquakes data only. Top panel: Observed data (black dots with error bars); ellipticity computed from the best value found (red line); ellipticity computed from models with cost function within 20 per cent from the best model. Bottom panels: Best model found (red solid line); models with cost function within 20 per cent from the best model (shadows of grey); model MAMBo-E by Berbellini et al. (2017) (black dashed line). Bottom right-hand panel: Zoom of the shallowest $6 \mathrm{~km}$.

Please note: Oxford University Press is not responsible for the content or functionality of any supporting materials supplied by the authors. Any queries (other than missing material) should be directed to the corresponding author for the article. 